\title{
Erratum to: Evaluating the complementary roles of an SJT and academic assessment for entry into clinical practice
}

\author{
Fran Cousans ${ }^{1,2} \cdot$ Fiona Patterson ${ }^{1,3} \cdot$ Helena Edwards ${ }^{1}$. \\ Kim Walker ${ }^{4,5} \cdot$ John C. McLachlan' $^{6}$ David Good $^{7}$
}

Published online: 2 March 2017

(C) Springer Science+Business Media Dordrecht 2017

\section{Erratum to: Adv in Health Sci Educ DOI 10.1007/s10459-017-9755-4}

Unfortunately an error was inadvertently introduced into Table 2 during the proofs procedure. The correct version is given below (Table 2).

The online version of the original article can be found under doi:10.1007/s10459-017-9755-4.

Fran Cousans

fcc9@le.ac.uk

Fiona Patterson

f.patterson@workpsychologygroup.com

Helena Edwards

h.edwards@workpsychologygroup.com

Kim Walker

kim.walker@nes.scot.nhs.uk

John C. McLachlan

j.c.mclachlan@durham.ac.uk

David Good

dg25@cam.ac.uk

1 Work Psychology Group, 27 Brunel Parkway, Pride Park, Derby DE24 8HR, UK

2 Occupational Psychology, Department of Neuroscience, Psychology and Behaviour, University of Leicester, Leicester LE1 7RH, UK

3 Department of Psychology, University of Cambridge, Downing Street, Cambridge CB2 3EB, UK

4 Scotland Foundation School Director, NHS Education for Scotland, Forest Grove House, Foresterhill Road, Aberdeen AB25 2ZP, Scotland, UK

5 UK Foundation Programme Office, St Chad's Court, 213 Hagley Road, Edgbaston, Birmingham B16 9RG, UK

6 Durham University, Holliday Building, Thornaby TS17 6BH, UK

7 Department of Psychology, University of Cambridge, Kings College, Downing Street, Cambridge CB2 3EB, UK 
Table 2 Descriptive statistics for predictor and outcome measures

\begin{tabular}{|c|c|c|c|c|c|c|c|c|c|c|}
\hline & \multicolumn{5}{|c|}{ High-scoring SJT group } & \multicolumn{5}{|c|}{ Low-scoring SJT group } \\
\hline & $\mathrm{N}$ & Min & Max & Mean & SD & $\mathrm{N}$ & Min & Max & Mean & $\mathrm{SD}$ \\
\hline \multicolumn{11}{|l|}{ Predictor variables } \\
\hline SJT score & 160 & 43.40 & 48.70 & 45.23 & 1.26 & 231 & 26.10 & 37.90 & 34.88 & 2.41 \\
\hline EPM score & 160 & 34.00 & 50.00 & 42.58 & 4.09 & 231 & 34.00 & 47.00 & 38.19 & 3.01 \\
\hline \multirow[t]{2}{*}{ Supervisors' overall score } & 160 & 2.70 & 6.00 & 5.05 & 0.73 & 231 & 1.15 & 6.00 & 4.64 & 0.95 \\
\hline & $\mathrm{N}$ & $\%$ & & & & $\mathrm{~N}$ & $\%$ & & & \\
\hline Remedial action & 2 & 1.3 & & & & 14 & 6.1 & & & \\
\hline No remedial action & 155 & 98.7 & & & & 215 & 93.9 & & & \\
\hline
\end{tabular}

